Revue de l'Institut des langues et cultures

d'Europe, Amérique, Afrique, Asie et Australie

$29 \mid 2017$

Les femmes en Russie : parcours, mythes et représentations

Katerina Izmaïlova est-elle une femme libérée?
Ambivalences du féminin dans Lady Macbeth du
district de Mtsensk de Dmitri Chostakovitch (1934)

АМБИВАЛЕНТНОСТЬ ЖЕНСКОГО ОБРАЗА В ОПЕРЕ ДМИТРИЯ ШОСТАКОВИЧА ЛЕДИ

МАКБЕТ МЦЕНСКОГО УЕЗДА (1934)

Catherine Géry

(2) OpenEdition

Journals

Édition électronique

URL : http://journals.openedition.org/ilcea/4231

DOI : 10.4000/ilcea.4231

ISSN : 2101-0609

Éditeur

UGA Éditions/Université Grenoble Alpes

Édition imprimée

ISBN : 978-2-37747-007-5

ISSN : 1639-6073

Référence électronique

Catherine Géry, « Katerina Izmaillova est-elle une femme libérée? Ambivalences du féminin dans Lady Macbeth du district de Mtsensk de Dmitri Chostakovitch (1934) », ILCEA [En ligne], 29 | 2017, mis en ligne le 30 juin 2017, consulté le 01 mai 2019. URL : http://journals.openedition.org/ilcea/4231 ; DOI : 10.4000/ilcea.4231

Ce document a été généré automatiquement le 1 mai 2019.

(C) ILCEA 


\section{Katerina Izmaillova est-elle une femme libérée? Ambivalences du féminin dans Lady Macbeth du district de Mtsensk de Dmitri Chostakovitch (1934)}

АМБИВАЛЕНТНОСТЬ ЖЕНСКОГО ОБРАЗА В ОПЕРЕ ДМИТРИЯ ШОСТАКОВИЧА ЛЕДИ МАКБЕТ МЦЕНСКОГО УЕЗДА (1934)

\section{Catherine Géry}

1 Caractérisé à la fois par la reconnaissance des pulsions et par leur répression, le XIX ${ }^{\mathrm{e}}$ siècle russe et européen a fréquemment conjugué le sexe et le meurtre, assimilé la sexualité féminine au démonisme et durablement associé les femmes à l'hystérie. Pour asseoir leurs visées déterministes, les écrivains ont convoqué et recyclé les grandes figures de meurtrières et les débauchées célèbres — Lilith, Médée, Salomé, Messaline ou Lady Macbeth - toutes susceptibles de créer « la terreur et la pitié », une terreur et une pitié qui ne s'expriment plus devant les désordres tragiques de la psyché, l'horreur des crimes de sang ou les troubles de la lignée, mais dont l'objet est désormais plus profondément enfoui dans les couches non conscientisées de l'individu. Cet objet innommé, omniprésent et obsédant, c'est une sexualité féminine "primitive ", qui se situe aux marges des grandes structures de la civilisation et renvoie la féminité à une animalité éminemment dangereuse pour la rationalité masculine ; et ceci d'autant plus que dans la seconde moitié du siècle, les premiers mouvements d'émancipation des femmes viennent menacer les bastions masculins multiséculaires qu'étaient la science et les arts ${ }^{1}$.

2 Le petit roman (ou grande nouvelle) de Nikolaï Leskov intitulé Lady Macbeth du district de Mtsensk est emblématique des peurs qu'ont pu susciter chez certains auteurs les volontés émancipatrices qui ont accompagné le courant nihiliste dans les années 1860 en Russie. 
Lady Macbeth est de bout en bout un texte paradoxal, faussement naïf, qui révèle l'image littéraire d'une sexualité féminine criminelle sur le fond des premiers mouvements de libération de la femme, tout en brisant l'interdit de représentation de la sexualité qui pèse sur toute la littérature russe savante depuis ses origines byzantines. Katerina Lvovna Izmaïlova, la «Lady Macbeth » de Leskov, est en proie à une passion sexuelle privée des freins de la morale commune et des convenances sociales, qui la pousse à commettre une série de crimes toujours plus atroces: elle empoisonne son beau-père avec des champignons, étrangle son mari avec l'aide de son amant, étouffe l'enfant qui doit hériter du domaine et noie une de ses rivales lors de la marche qui la conduit au bagne en Sibérie ; elle finira dans les ténèbres de la possession et sera durement châtiée par une mort ignominieuse, seule rétribution possible à cet ébranlement massif des lois humaines que sa conduite a provoqué.

3 En 1934, le compositeur Dmitri Chostakovitch proposait au public soviétique son adaptation de la nouvelle de Leskov (Lady Macbeth du district de Mtsensk, opus 29). Le succès national et international de l'opéra fut immense avant qu'il ne soit jeté en pâture aux censeurs : en janvier 1936, un collaborateur anonyme de la Pravda dans lequel d'aucuns ont cru reconnaître Staline lui-même (il s'agissait sans doute de David Zaslavski,

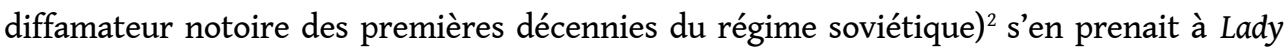
Macbeth avec une violence inouïe, à l'intérieur d'un article intitulé « Le chaos remplace la musique » [сумБур вмЕсто мУзыки] :

\begin{tabular}{|c|c|}
\hline Le chaos remplace la musique & СУМБУР ВМЕСТО МУЗЫКИ \\
\hline $\begin{array}{l}\text { Un flux de sons chaotiques et } \\
\text { volontairement disharmonieux frappe } \\
\text { l'auditeur de stupeur dès la toute première } \\
\text { minute de l'opéra. Les bribes d'une } \\
\text { mélodie, les germes d'une phrase musicale } \\
\text { se noient, s'échappent, disparaissent de } \\
\text { nouveau dans le fracas, les grincements et } \\
\text { les glapissements. Suivre cette } \\
\text { «musique " est difficile, s'en souvenir } \\
\text { impossible. } \\
\text { [...] Au moment où notre critique (dont la } \\
\text { critique musicale) ne jure que par le } \\
\text { réalisme socialiste, la scène nous présente } \\
\text { en l'œuvre de chostakovitch le } \\
\text { naturalisme le plus grossier [...]. La } \\
\text { musique glousse, huche, halète, suffoque } \\
\text { afin de représenter les scènes d'amour de } \\
\text { la façon la plus naturelle qui soit. Et } \\
\text { «l'amour » est étalé dans tout l'opéra sous } \\
\text { sa forme la plus vulgaire. }\end{array}$ & $\begin{array}{l}\text { СЛУШАТЕЛЯ С ПЕРВОЙ ЖЕ МИНУТЫ ОШАРАШИВАЕТ } \\
\text { В ОПЕРЕ НАРОЧИТО НЕСТРОЙНЫЙ, СУМБУРНЫЙ } \\
\text { ПОТОК ЗВУКОВ. ОБРЫВКИ МЕЛОДИИ, ЗАЧАТКИ } \\
\text { МУЗЫКАЛЬНОЙ ФРАЗЫ ТОНУТ, ВЫРЫВАЮТСЯ, } \\
\text { СНОВА ИСЧЕЗАЮТ В ГРОХОТЕ, СКРЕЖЕТЕ И ВИЗГЕ. } \\
\text { СЛЕДИТЬ ЗА ЭТОЙ МУЗЫКОЙ» ТРУДНО, } \\
\text { ЗАПОМНИТЬ ЕЕ НЕВОЗМОЖНО. } \\
\text { <... В ТО ВРЕМЯ, КАК НАША КРИТИКА - В ТОМ } \\
\text { ЧИСЛЕ И МУЗЫКАЛЬНАЯ - КЛЯНЕТСЯ ИМЕНЕМ } \\
\text { СОЦИАЛИСТИЧЕСКОГО РЕАЛИЗМА, СЦЕНА } \\
\text { ПРЕПОДНОСИТ НАМ В ТВОРЕНИИ ШОСТАКОВИЧА } \\
\text { ГРУБЕЙШИЙ НАТУРАЛИЗМ. <..> МУЗЫКА КРЯКАЕТ, } \\
\text { УХАЕТ, ПЫХТИТ, ЗАДЫХАЕТС, ЧТОБЫ КАК МОЖНО } \\
\text { НАТУРАЛЬНЕЕ ИЗОБРАЗИТЬ ЛЮБОВНЫЕ СЦЕНЫ. И } \\
\text { «ЛЮБОВЬ» РАЗМАЗАНА ВО ВСЕЙ ОПЕРЕ В САМОЙ } \\
\text { ВУЛЬГАРНОЙ ФОРМЕ } .\end{array}$ \\
\hline
\end{tabular}

4 Accusé de naturalisme (entendre de vulgarité et d'obscénité) par les censeurs, l'opéra de Chostakovitch s'est aussi trouvé pris au cœur de la campagne contre le formalisme, autrement dit contre tout ce qui relève d'un art complexe et surtout impropre à servir les objectifs politiques du pouvoir dans le domaine de la culture. Pourtant, le compositeur ne 
pensait pas faire œuvre d'hérétique en adaptant Lady Macbeth du district de Mtsensk au début des années 1930, bien au contraire.

Les raisons du choix par Chostakovitch de la nouvelle de Leskov sont diverses, et il est difficile de privilégier l'une ou l'autre hypothèse parmi celles que je vais exposer. Tout d'abord, le compositeur était familier des adaptations des textes de la littérature pour l'opéra. Il venait de finir Le Nez d'après Gogol, une expérience "avant-gardiste » influencée par l'expressionnisme allemand, menée conjointement avec Alexandre Preis et Evgueni Zamiatine qui avait travaillé avec lui sur le livret. Il en était résulté une œuvre où la veine misogyne battait assez fort et où s'exprimait une véritable phobie des «femmes perfides ». L'idée de consacrer un opéra "goethéen" à une femme, fatale cette fois-ci, aurait été suggérée à Chostakovitch par le compositeur et musicologue Boris Assafiev, personnalité influente de la vie musicale soviétique des années $1930^{4}$. Chostakovitch connaissait et appréciait par ailleurs les œuvres de Leskov (qui n'était pas encore devenu l'auteur suspect de toutes les turpitudes réactionnaires qu'il serait quelques années plus tard) et il était proche des avant-gardes des années 1920 pour lesquelles Leskov était un maître. En 1926, le compositeur avait vu avec intérêt le film de Tcheslav Sabinski Katerina Izmailova ${ }^{5}$, une version « soviétisée » de Lady Macbeth qui se présentait comme une lecture sociale du texte de Leskov (une satire dénonciatrice), ce qui sera aussi la position de Chostakovitch dans son opéra. Enfin, on peut penser, à la suite du musicologue Solomon Volkov, que l'attention du compositeur a été attirée par l'édition de la nouvelle de Leskov illustrée par Boris Koustodiev, et que c'est ce petit volume, paru en 1930 aux Éditions des écrivains qui l'a définitivement convaincu d'adapter Lady Macbeth du district de Mtsensk (Volkov, 2004 : 152).

6 Solomon Volkov affirme qu'à côté des illustrations «légitimes » ou «montrables » du récit de Leskov, Koustodiev «avait dessiné d'innombrables variantes érotiques [...] qui n'étaient pas destinées à la publication. À sa mort, craignant les perquisitions, la famille de Koustodiev s'est empressée de détruire ces dessins » (ibid.). L'information livrée par Volkov est de poids, car ce dernier suppose que Chostakovitch a vu ces esquisses et que l'érotisme appuyé de son opéra en est directement redevable.

7 Cet érotisme a été prudemment passé sous silence dans les premières critiques de Lady Macbeth du district de Mtsensk avant 1936. Seuls Eisenstein et Prokofiev ont souligné, le premier en public et le second en privé, l'extrême tension érotique de l'œuvre ${ }^{6}$. Les premières représentations de l'opéra en janvier 1934, celle de Samuel Samosud pour le Petit théâtre de Leningrad [Malyj leningradskij Teatr] dont il a également dirigé l'orchestration, et celle de Vladimir Nemirovitch-Dantchenko pour le Théâtre musical de Moscou [Muzykal'nyj moskovskij Teatr], se montraient très sensibles à la dimension pansexualiste de la nouvelle de Leskov et se distinguaient par une accentuation du "moment érotique»: la scène d'amour entre Katerina et Sergueï était ainsi particulièrement appuyée et exhibée, elle se passait dans un lit géant devant la rampe de la scène (où le mari était tué quelques instants plus tard).

Martin Kušej, qui a récemment mis en scène Lady Macbeth pour le Nederlandse Opera d'Amsterdam (présentée à Paris à l'opéra Bastille en janvier 2009), a encore accru cette tension érotique. La nudité parfois à la limite de la vulgarité, l'impression très forte de corporéité qui se dégage de son interprétation sont symptomatiques d'une lecture décomplexée et « désengagée » de l'opéra de Chostakovitch. 
9 En 2000, Dmitri Bertman a également signé pour le Gelikon-Opera de Moscou une version crue et violente de Lady Macbeth, dont le programme portait en exergue l'avertissement suivant : « Pour adultes uniquement ! »

10 Ces deux mises en scènes contemporaines, celle de Kušej et celle de Bertman, renvoient à une violence physique qui n'est absente ni du livret original, ni de la partition. Je prendrai pour exemple le passage où, dans la nouvelle de Leskov, Aksinia, la servante de Katerina, se fait peser par les commis, un moment que Chostakovitch a interprété comme une métaphore de l'acte sexuel, transformant l'épisode en scène de viol collectif et parachevant en quelque sorte le geste interrompu de Leskov. Cette scène sera d'ailleurs en partie expurgée de la deuxième version de l'opéra qui verra le jour au début des années 1960 sous le titre de Katerina Izmailova.

11 Voici les deux épisodes, le premier tel qu'on peut le lire chez Leskov, et le second dans sa transposition par Chostakovitch. 


\section{LESKOV :}

On respire si largement dans la cour baignée de clarté, et dans les galeries près des entrepôts résonnent de gros rires joyeux.

«Et de quoi donc on se réjouit comme ça? a demandé Katerina Lvovna aux commis de son beau-père.

- Ben voilà, Katerina Lvovna, not' daronne, on a pesé une truie vivante, lui a répondu un vieux commis.

- Quelle truie?

- La truie Aksinia, celle qui a enfanté un fils, Vassili, et qui ne nous a pas invités au baptême ", a expliqué en riant un beau gars au visage hardi, encadré par des boucles noires comme de la poix et une barbe qui avait encore à peine poussé.

La grosse trogne rougeaude de la cuisinière Aksinia a paru en cet instant hors du tonneau suspendu au fléau de la balance.

"Démons, diables repus! jurait la cuisinière en s'efforçant de saisir la barre de fer du fléau pour sortir du tonneau qui oscillait sous elle.

- Elle pèse bien ses huit pouds à jeun ; une fois mangé son banneau d'foin, tous nos poids n'y suffiront point » a repris le beau gars et, retournant le tonneau, il a renversé la cuisinière sur des sacs de tille stockés dans le charbon.

\section{CHOSTAKOVITCH - PREIS :}

(Acte I, deuxième tableau)

La cour. Les serviteurs de Zinovi s'amusent : ils ont enfermé Aksinia dans un tonneau sans fond ni couvercle et refusent de la libérer.

\section{AKSINIA}

Aïe, aïe, aïe, effronté, arrête de me pincer,

Aïe ça fait mal, aïe ça fait mal, Enlève tes mains de là,

Diable effronté, bas les pattes, Diable dégoûtant, diable dégoûtant, Ôte-toi de là! Ah salaud, aïe, aiie, aïe!

Salaud, salaud, salaud, salaud ! Ah quel salaud, ah quel salaud! Aïe ça fait mal, aïe ça fait mal !

\section{LE BALOURD MITEUX}

Ah le joli rossignol !

Tiens que je te palpe, que je te palpe!

Que je te serre! encore!

Quel téton, ah, quel téton,

Ah le téton, ah le téton, ah le téton!

Ah que c'est lisse !

LE PORTIER

La truie chante comme un

rossignol,

Fouille, fouille, mieux que ça !

Etc. 


\section{ЛЕСКОВ:}

НА ДВОРЕ ТАК СВЕТЛО И КРЕПКО ДЫШИТСЯ, А НА ГАЛЕРЕЕ У АМБАРОВ ТАКОЙ ХОХОТ ВЕСЕЛЫЙ СТОИТ.

- ЧЕГО ЭТО ВЫ ТАК РАДУЕТЕСЬ? - СПРОСИЛА КАТЕРИНА ЛЬВОВНА СВЕКРОВЫХ ПРИКАЗЧИКОВ.

- А ВОТ, МАТУШКА КАТЕРИНА ИЛЬВОВНА, СВИНЬЮ ЖИВУЮ ВЕШАЛИ, - ОТВЕЧАЛ ЕЙ СТАРЫЙ ПРИКАЗЧИК.

- КАКУЮ СВИНЬЮ?

- А ВОТ СВИНЬЮ АКСИНЬЮ, ЧТО РОДИЛА СЫНА ВАСИЛЬЯ ДА НЕ ПОЗВАЛА НАС НА КРЕСТИНЫ, - СМЕЛО И ВЕСЕЛО РАССКАЗЫВАЛ МОЛОДЕЦ С ДЕРЗКИМ КРАСИВЫМ ЛИЦОМ, ОБРАМЛЕННЫМ ЧЕРНЫМИ КАК СМОЛЬ КУДРЯМИ И ЕДВА ПРОБИВАЮЩЕЙСЯ БОРОДКОЙ.

ИЗ МУЧНОЙ КАДИ, ПРИВЕШЕННОЙ К ВЕСОВОМУ КОРОМЫСЛУ, В ЭТУ МИНУТУ ВЫГЛЯНУЛА ТОЛСТАЯ РОЖА РУМЯНОЙ КУХАРКИ АКСИНЬИ.

- ЧЕРТИ, ДЬЯВОЛЫ ГЛАДКИЕ, - РУГАЛАСЬ КУХАРКА, СТАРАЯСЬ СХВАТИТЬСЯ ЗА ЖЕЛЕЗНОЕ КОРОМЫСЛО И ВЫЛЕЗТЬ ИЗ РАСКАЧИВАЮЩЕЙСЯ КАДИ.

- ВОСЕМЬ ПУДОВ ДО ОБЕДА ТЯНЕТ, А ПИХТЕРЬ СЕНА СЪЕСТ, ТАК И ГИРЬ НЕДОСТАНЕТ, - ОПЯТЬ ОБЪЯСНИЛ КРАСИВЫЙ МОЛОДЕЦ И, ПОВЕРНУВ КАДЬ, ВЫБРОСИЛ КУХАРКУ НА СЛОЖЕННОЕ В УГЛЕ КУЛЬЕ.

\section{ШОСТАКОВИЧ - ПРЕЙС:}

КАРТИНА ВТОРАЯ

(ДВОР. РАБОТНИКИ ИЗМАЙЛОВЫХ, ПОСАДИВ АКСИНЬЮ В БОЧКУ БЕЗ ДНИЩА, КАТАЮТ БОЧКУ ПО ДВОРУ, НЕ ДАВАЯ АКСИНЬЕ ВЫБРАТЬСЯ ИЗ НЕЁ.)

АксиньЯ. АЙ, АЙ. АЙ, АЙ!

ЗАДРИПАННЫЙ МУЖИЧОНКА. ПРЯМО СОЛОВУШКА! А НУ, ПОЩУПАЙ! А НУ, ПОЩУПАЙ!

ДВОРНИК. СВИНЬЯ СОЛОВЬЁМ ЗАЛИВАЕТСЯ!

СТАРШИЙ ПРИКАЗЧИК. ОГО! ЖИРУто, жИРу!

РАБОТНИКИ. НУ И ГОЛОСОК! НУ И голосок! ну и голосок!

АКСИНЬЯ. АЙ, БЕССТЫЖИЙ! ОЙ, НЕ щипли! АЙ, Больно! АЙ, Больно! ИШЬ ТЫ, КУДА ПОЛЕЗ.

МУЖИЧОНКА. ПРИЖМИ... ЕЩЁ!

ДВОРНИК. ЛОВЧЕЕ,... ПОШАРЬ, ШАРЬ!...

ПРИКАЗЧИК. ЕЩЁ! ТАК, ТАК! А ЕЩЁ! $\cdots$

РАБОТНИКИ. НУ И ГОЛОСОК! ХА, ХА, ХА! НУ И ГОЛОСОК, НУ И ГОЛОсок!

АКСИНЬЯ. БЕССТЫЖИЙ чЁРТ, КУДА ПОЛЕЗ! ПАРШИВЫЙ ЧЁРТ, ПАРШИВЫЙ ЧЁРТ, НЕ ЛЕЗЬ! АХ, чЁРТ! ПОшЁл! АЙ... СволОчь!

МУЖИЧОНКА. ВОТ ТАК ВЫМЯ, НУ И ВЫмЯ. АЙ, ВЫМЯ, ОЙ ВЫмЯ. АЙ, ГЛАДКОЕ, ЖМИ, ЖМИ, ЖМИ, ЖМИ! 
12 Tous les moyens musicaux expriment ici la violence sexuelle: saturation de l'espace sonore, enchaînement continu des motifs, rapidité du tempo, montée chromatique des cris aboutissant à un fortissimo de l'orchestre, suivi d'un brusque silence. Plaisir et violence ont partie liée dans l'opéra de Chostakovitch, comme on peut s'en convaincre dans une autre scène tout aussi cruelle, et même à l'extrême limite de la perversité, où le beau-père de Katerina Lvovna fouette Sergueï sous les yeux de la jeune femme, "juste pour le plaisir ", alors que la musique atteint un paroxysme de violence expressionniste.

Ces exemples ne peuvent toutefois cacher le fait que tout en en révélant les contenus érotiques latents du texte source et en revendiquant une couleur sexuelle soutenue par un discours musical d'une forte tension dramatique, l'opéra de Chostakovitch réduit singulièrement la portée subversive de la figure de femme créée par Leskov. Certes, il rompt de façon spectaculaire avec la tradition de l'opéra russe, héritée du XIX ${ }^{\mathrm{e}}$ siècle, de figures féminines patientes, douces et aimantes ou portées au sacrifice de soi en mettant en scène une nature passionnée et volontaire, presque "sauvage »; mais il ne fait pas pour autant de Katerina Lvovna une femme dominée par ses seules pulsions, une créature inquiétante et dangereuse (ce qu'elle était de toute évidence chez Leskov), et encore moins une figure du mal. Bien au contraire, il la transforme en une victime de l'ordre social, réduisant ses meurtres à "une révolte contre son entourage ${ }^{8}$ ", allant jusqu'à parler du « destin d'une femme brillante, remarquable, intelligente, qui meurt dans des conditions épouvantables dans la Russie bourgeoise et féodale d'avant la Révolution ${ }^{9}$ »!) et «dans un cadre de vie familial morbide et oppressant ${ }^{10}$ ", incarné dans l'opéra par le beau-père, Boris Timofeïevitch, un digne représentant de l'ancien ordre patriarcal, phallocrate et tyrannique.

Le but affirmé de Chostakovitch est donc de " justifier » Katerina Lvovna, de la disculper et peut-être même de la racheter. Mais dans le même temps, le compositeur était parfaitement conscient de ce que son traitement du récit de Leskov allait à l'encontre du sens profond du texte : «J'ai interverti les places de la victime et du bourreau » aurait-il affirmé au cours d'une de ses interviews.

D'une certaine façon, Chostakovitch a refaçonné et en quelque sorte «normalisé » l'héroïne de Leskov sur le modèle d'une autre femme adultère qui lui était contemporaine, la Katia Kabanova de L'Orage d'Ostrovski et de son interprétation par Leoš Janaček dans son opéra de 1921. Dans Káta Kabanová, Janaček a surtout centré son propos sur la dénonciation des comportements et des institutions. Un seul moment de l'opéra rend compte de l'état quasiment pathologique de Káta: il s'agit de sa "confession » (Acte I, second tableau), quand la musique même souligne que la démarcation entre l'extase religieuse et l'extase sexuelle est extrêmement ténue, alors que Káta raconte à son amie quels étranges désirs s'emparent d'elle ( “C'est comme si le diable murmurait à mon oreille des choses mauvaises » - «Jakoby mi d'abel našeptával / a samé takové nedobré věci ») ${ }^{11}$.

Afin de rendre plus vraisemblable la métamorphose de la meurtrière en victime et lui ôter le statut peu recommandable de «tueuse en série » qu'elle avait acquis chez Leskov, Chostakovitch et son compère, le dramaturge Alexandre Preis, ont supprimé l'assassinat forcément odieux et difficilement justifiable aux yeux de la morale commune du neveu de Katerina Lvovna, car, comme l'affirmait laconiquement le compositeur, « le meurtre d'un 
enfant fait toujours mauvaise impression ». Dans la foulée, Chostakovitch a également rajouté plusieurs scènes au texte de Leskov, toutes de tonalité grotesque et satirique, justifiant par là même l'indication de genre donnée dans le sous-titre de l'opéra: «tragédie-satire ». Dans Lady Macbeth, Katerina porte seule le tragique, et pour la caractériser, Chostakovitch a souvent fait usage d'une musique lyrique, un « rayon de lumière » qui jaillit du « royaume des ténèbres » pour reprendre l'expression célèbre de Dobrolioubov à propos de Katia Kabanova chez Ostrovski. Le langage musical renforce le discours idéologique : les arias lyriques et les cantilènes (brèves mélodies lyriques) sont réservés à la seule Katerina ${ }^{12}$. Pour les autres personnages, pervertis ou pervers, goujats, tyrans ou impuissants, ce sont les ruptures, les dissonances et les récitatifs (chants librement déclamés) qui dominent. La satire a pour objet l'ordre régnant, incarné par les hommes; la tragédie est celle de la femme qui se révolte en vain contre cet ordre.

C'est sans doute pourquoi à aucun moment la revendication par Katerina de son droit à la jouissance sexuelle n'encourt la réprobation morale de l'auteur. L'opéra ne fonctionne d'ailleurs pas sur le mécanisme de censure et de refoulement/défoulement face à la sexualité féminine qui caractérisait le texte de Leskov; dans la même optique, la sexualité n'y fait l'objet d'aucune transposition symbolique. Chostakovitch mentionne directement l'impuissance du mari, là où Leskov ne faisait que la suggérer, et Katerina souligne très crûment les insuffisances physiques et sexuelles de son mari (« une souche, un rondin, chétif, froid comme un poisson... »). Chostakovitch présente le beau-père sous les traits d'un vieillard ouvertement concupiscent, plein de désir pour sa bru qu'à la place de son benêt de fils, il saurait bien contenter et peut-être même engrosser, mettant fin à la malédiction de la stérilité. L'opéra montre également Sergueï comme un homme à l'avidité sexuelle brutale (il viole la cuisinière, force sa patronne et finit par briguer les faveurs d'une prostituée), là où Leskov en avait fait une presque victime des appétits de Katerina Lvovna. Enfin, chez Leskov, le meurtre s'inscrivait dans la continuité des débordements sexuels, il en était la suite logique ; chez Chostakovitch, c'est le résultat de la volonté d'émancipation de l'héroïne et un exutoire aux humiliations subies. On assiste ainsi dans son opéra à la résurgence d'une interprétation de la nouvelle de Leskov fortement inspirée par la pensée radicale des années 1860. Chostakovitch s'en est d'ailleurs ouvertement recommandé, et je rappellerai ici que le compositeur a grandi dans une famille révolutionnaire sous l'influence des idées de Tchernychevski, dont le Que faire ? avait gouverné, dit-il, toute la vie de sa grand-mère maternelle ; le grand-père de Chostakovitch avait quant à lui été impliqué dans l'évasion manquée de Tchernychevski lorsqu'il était au bagne. Aussi Lady Macbeth est-il un opéra politique, un opéra de la désaliénation où la critique des pouvoirs et des institutions est manifeste, et qui rappelle la charge satirique du Wozzeck d'Alban Berg (1925), un des modèles dramatiques et musicaux avoués de Chostakovitch. L'influence de Berg se retrouve aussi au niveau des thèmes de la compassion sociale et de la frustration sexuelle des femmes.

Avec Lady Macbeth, Chostakovitch signe une œuvre d'un féminisme militant mais politiquement décalé, comme le prouve le terrible destin de l'opéra. Les thèmes de l'émancipation sexuelle des femmes avaient été fort à la mode au cours de la décennie précédente : on sait l'intérêt que portait à ces questions Alexandra Kollontaï (1978), qu'un débat célèbre sur l'amour libre avait opposée à Lénine. Chostakovitch avait été encouragé à s'exprimer sur le sujet de la sexualité féminine par ses discussions avec son ami, le musicologue Ivan Sollertinski, qui regrettait qu'il n'y eût dans l'opéra russe aucun exemple semblable à Carmen ou Wozzeck (Volkov, 1980 : 149). 
19 Si l'opéra restait quelque peu à la traîne, au théâtre on pouvait voir en revanche au cours des années 1920 des spectacles sur divers thèmes liés à la libération des femmes ou à la sexualité. En 1926, la pièce de Sergueï Trétiakov Je veux un enfant! [ХОчУ РЕБЁНКА!] abordait avec une grande crudité les problèmes de la reproduction (Vsevolod Meyerhold a tenté de monter cette pièce qui fut néanmoins interdite par les autorités pour cause d'indécence). Au cinéma, le film très populaire d'Abram Room Troisième rue des PetitsBourgeois [ТРЕТЬЯ МЕЩАНСКАЯ] mettait aux prises une jeune femme décidée et deux hommes veules, permettant au thème «bourgeois » du ménage à trois de déboucher sur la question d'une nouvelle morale, socialiste et émancipatrice. Chostakovitch s'est donc laissé porter (avec un retard tragique de quelques années) par l'atmosphère des années 1920, une atmosphère somme toute assez proche de celle qui régnait dans les cercles progressistes russes au cours des années 1860, quand Leskov rédigeait sa Lady Macbeth. Le parallèle est saisissant, jusque dans la conception de l'amour comme une donnée physiologique commune aux nihilistes comme à «l'art de gauche» soviétique. Dans cet art dit de gauche, la sexualité est envisagée essentiellement sous l'angle physiologique, comme une technique de reproduction humaine, elle est assimilée à la procréation; dans la pièce de Trétiakov que j'ai évoquée plus haut, la préoccupation principale de Milda, ouvrière de choc et incarnation de l'élite prolétarienne féminine, est de se trouver un solide mâle reproducteur, de bonne origine sociale, destiné à servir la construction du socialisme...

Mais les quelques années qui séparaient le film de Room ou la pièce de Trétiakov de la sortie de l'opéra de Chostakovitch (bien qu'achevé en 1932, il n'a été monté qu'en 1934) ont créé une dissonance fatale : au milieu des années 1930, ce ne sont plus les débats sur la sexualité et encore moins l'affirmation de la liberté sexuelle qui sont d'actualité, mais la traque de l'outrage aux bonnes mœurs, et les scènes de lit ont durablement disparu des théâtres comme des écrans de cinéma : on peut voir ce qui semble être la dernière sous Staline dans le film d'Albert Gendelstein Amour et haine sorti tardivement en 1935 ; la musique du film était signée... Chostakovitch.

21 Aussi, il semblerait bien que Lady Macbeth du district de Mtsensk soit une ceuvre des années 1920 malencontreusement égarée dans les années 1930, les années de la normalisation sexuelle, de l'interdiction de la psychanalyse, des mesures prises en faveur du renforcement de la famille (comme en témoigne le nouveau Code du mariage et de la famille de 1936), de l'héroïsation de la figure maternelle. Comme l'affirme Piotr Kaminski, «nous sommes loin des utopies émancipatrices et féministes d'Alexandra Kollontaï qui eût sans doute bien aimé cet opéra. Le pouvoir est de nouveau à Boris Timofeïevitch » (1991: 104), c'est-à-dire au vieil ordre patriarcal et phallocrate qui régnait sans partage chez Leskov.

J'aimerais pour conclure tisser un nouveau lien entre l'opéra et sa source littéraire, qui sont deux œuvres qui fonctionnent selon les lois de la catharsis et de la violence purificatrice (j'emploie ici le terme de « catharsis » dans ses deux acceptions principales : au sens aristotélicien de "purgation des passions par la terreur et la pitié éprouvées devant une destinée tragique", et au sens psychanalytique de libération des traumatismes refoulés). Chez Leskov, c'est de la purgation des passions de l'auteur qu'il s'agit, de la complexité de son rapport à la jouissance sexuelle revendiquée par son héroïne et, à travers elle, par toute une génération de femmes. Chez Chostakovitch, le phénomène de catharsis relèverait plutôt d'une douloureuse prise de conscience historique : le personnage de Katerina, qui apparaît dans son opéra sous le double visage 
du bourreau et de la victime, peut être envisagé comme l'émanation culturelle d'une époque paradoxale qui, face à la montée des violences totalitaires, reste partagée entre terreur et fascination. Il me semble ici possible d'appliquer à l'opéra de Chostakovitch les théories développées par Alexandre Jolkovski sur l'ambivalence constitutive aux grandes œuvres nées sous le stalinisme (Žolkovskij, $1999: 308-311)^{13}$. Comme tant d'autres artistes de sa génération, Chostakovitch a fait montre d'une attirance certaine pour le stalinisme et a participé à la construction de la culture soviétique dominante. Cette attirance à la fois mêlée de peur et de défiance, cette participation ambiguë aux projets et aux postulats du stalinisme sont à l'origine du caractère équivoque et de l'instabilité de son opéra. Les mécanismes de contournement des interdits et d'intégration de ces mêmes interdits, d'obéissance à la doxa et de contestation, parfois involontaire, de ses préceptes, d'amour ambivalent de l'ordre et de défiance envers les autorités qui l'incarnent, de canonisation des modèles et de leur dégradation font de Lady Macbeth une œuvre authentique de la période stalinienne, qui reflète en profondeur la réalité de l'époque, avec ses peurs, ses séductions et ses stratégies.

La violence extrême qui caractérise Lady Macbeth du district de Mtsensk n'est donc pas uniquement (ni même essentiellement) celle des rapports sociaux dans la société tsariste, dénoncés par un jeune auteur soucieux de donner des gages de bonne pensée au pouvoir soviétique. Et en nous livrant, après le Wozzeck d'Alban Berg, son propre «opéra de la compassion sociale ", Chostakovitch, qui n'a jamais été entièrement convaincu de la validité de l'orientation idéologique qu'il avait donnée à Lady Macbeth, a peut-être essayé de se purger d'un des péchés parmi les plus inexcusables en URSS : le péché de doute.

\section{BIBLIOGRAPHIE}

ASAF'EV Boris (1957), Izbrannye trudy [Travaux choisis], Moscou : AN SSSR.

BRÈQUE Jean-Michel (1991), « Une Lady Macbeth modérément shakespearienne », L'Avant-Scène Opéra, 141.

DeRMOnCOURT Bertrand (2006), Dimitri Chostakovitch, Arles : Actes Sud.

KAMINSKI Piotr (1991), « Discographie », L'Avant-Scène Opéra, 141.

Kollontaï Alexandra (1978), Conférence sur la libération des femmes, Paris : éditions La Brèche.

LISCHKE André (1991), « L'opéra et la censure. Lady Macbeth face à Katerina Ismailova », L'AvantScène Opéra, 141, 13-23.

VoLKov Solomon (1980), Témoignage. Les mémoires de Dimitri Chostakovitch, Paris : Albin Michel.

VolKov Solomon (2004), «Stalin i Šostakovič: slučaj Ledi Makbet Mtsenskogo uezda » [Staline et Chostakovitch : le cas de Lady Macbeth du district de Mtsensk], Znamja [L'Étendard], 8.

VolKov Solomon (2005), Chostakovitch et Staline, Paris : Éditions du Rocher.

Žolkovsкij A. (1999), M. Zoščenko. Poètika nedoverija [M. Zochtchenko. Une poétique de la défiance], Moscou : Škola « Jazyki russkoj Kul'tury». 


\section{NOTES}

1. On a eu récemment un témoignage de la fascination du $\mathrm{XIX}^{\mathrm{e}}$ siècle pour les femmes « dangereuses » avec l'exposition L'Ange du bizarre. Le romantisme noir, de Goya à Max Ernst, qui s'est tenue à Paris au musée d'Orsay du 5 mars au 9 juin 2013, et qui tentait d'appliquer à l'histoire de l'art les catégories définies par Mario Praz dans son célèbre ouvrage La Chair, la mort et le diable dans la littérature du XIX ${ }^{e}$ siècle. Le romantisme noir, Paris, Denoël, coll. « Tel », 1977.

2. S. Volkov, Shostakovich and Stalin, New York, Alfred A. Knopf, 2004 ; l'ouvrage est paru en russe à Moscou aux éditions Èksmo la même année sous le titre Šostakovič $i$ Stalin: xudožnik $i$ car [Chostakovitch et Staline : l'Artiste et le Tsar] et en français en 2005 sous le titre Chostakovitch et Staline (Paris, Éditions du Rocher).

3. «Sumbur vmesto muzyki » [Le bruit remplace la musique], Pravda, 28 janvier 1936. Texte consulté en ligne le 17 janvier 2017 sur <www.theremin.ru/archive/sovok/sumbur.htm>.

4. Lequel parlera cependant après 1936 de la «sensualité cyniquement exhibitionniste » de l'opéra de Chostakovitch et du "penchant cruel et grossier » du compositeur pour "des états pathologiques » (Asaf'ev, 1957, t. $5: 118$ ).

5. C'est ce qu'affirme Lischke (1991: 13).

6. Cités par Volkov (2005: 122).

7. Le spectacle a été repris avec succès en 2010 et 2011.

8. Dmitri Chostakovitch, Comment j'ai conçu Lady Macbeth, Moscou, 1935, cité par Brèque (1991: 10).

9. Sovetskoe Iskusstvo [L'art soviétique], 16 octobre 1932.

10. Sovetskoe Iskusstvo [L'art soviétique], 14 décembre 1933.

11. Un épisode fait d'ailleurs se rejoindre les trois œuvres, celle d'Ostrovski, de Janaček et de Chostakovitch : il s'agit de la scène des adieux de Zinovi à Katerina au $1^{\mathrm{er}}$ tableau de Lady Macbeth du district de Mtsensk avec le serment de fidélité et d'allégeance arraché de force par le vieux Boris, une scène que Chostakovitch a très probablement empruntée à L'Orage d'Ostrovski et qui rappelle furieusement dans son traitement par le compositeur russe la scène correspondante chez Janaček.

12. Cf. la plainte lyrique de Katerina au premier tableau, l'aria fameux qui ouvre le troisième tableau et l'aria final, quand Katerina chante son désir de mort, d'abolition dans les eaux noires d'un lac au cœur de la forêt.

13. Le biographe de Chostakovitch, Bertrand Dermoncourt (2006), analyse dans le même sens l'œuvre et la démarche du compositeur (une œuvre et une démarche fondées sur l'ambiguïté) pour expliquer l'extraordinaire longévité d'un créateur qui a pu concilier une véritable carrière sous un régime totalitaire et l'édification d'une œuvre personnelle.

\section{RÉSUMÉS}

Le petit roman de Nikolaï Leskov Lady Macbeth du district de Mtsensk (1865) est un texte paradoxal, faussement naïf, habité par la peur et le désir, qui révèle curieusement l'image d'une sexualité féminine criminelle sur le fond des premiers mouvements de libération de la femme dans les 
années 1860 en Russie, tout en brisant l'interdit de représentation de la sexualité qui pèse sur toute la littérature russe savante depuis ses origines byzantines. Katerina Izmaïlova, la « Lady Macbeth » de Leskov, est en proie à une passion sexuelle privée des freins de la morale commune et des convenances sociales qui la pousse à commettre une série de crimes toujours plus atroces ; elle finira dans les ténèbres de la possession et sera durement châtiée par une mort ignominieuse, seule rétribution possible à l'ébranlement massif des lois humaines que sa conduite a provoqué.

À partir de cette figure de femme dominée par ses seules pulsions et qui mettait gravement en danger l'ordre rationnel masculin, Dmitri Chostakovitch a réalisé un opéra de la désaliénation et de la compassion sociale, faisant subir de sérieuses transformations au personnage de Katerina Izmaïlova : l'héroïne de Leskov devient ici une "meurtrière innocente ", une victime tragique de la violence et de l'oppression qui caractérisaient l'ordre moral et social mortifère de « la Russie bourgeoise et féodale" d'avant la Révolution. Chez Leskov, le meurtre s'inscrivait dans la continuité des débordements sexuels; chez Chostakovitch, qui ne fait peser aucun jugement moral sur la revendication par Katerina de son droit à la jouissance sexuelle, c'est le résultat d'une volonté d'émancipation et un exutoire aux humiliations subies.

Cependant, l'œuvre de Chostakovitch est profondément ambivalente, et la violence extrême qui caractérise tout l'opéra (dans ses motifs sociaux comme dans sa configuration musicale) n'est pas uniquement celle des rapports et des comportements dans la société russe patriarcale et phallocrate de la seconde moitié du XIX ${ }^{e}$ siècle, dénoncée par un jeune auteur soucieux de donner des gages de bonne pensée au pouvoir soviétique. Katerina, qui apparaît dans Lady Macbeth du district de Mtsensk sous le double visage du bourreau et de la victime, peut également être envisagée comme l'émanation culturelle d'un moment historique («l'épisode stalinien») qui, face à la montée des violences totalitaires, reste partagé entre la terreur et la fascination.

ПОВЕСТЬ Н.С. ЛЕСКОВА ЛЕДИ МАКБЕТ МЦЕНСКОГО УЕЗДА (1865) - ПАРАДОКСАЛЬНЫЙ ТЕКСТ, КОТОРЫЙ С ОДНОЙ СТОРОНЫ ПРЕДСТАВЛЯЕТ ОБРАЗ ЖЕНСКОЙ ПРЕСТУПНОЙ СЕКСУАЛЬНОСТИ В ТОТ МОМЕНТ, КОГДА В РОССИИ 60-ЫХ ГОДОВ ХІХ ВЕКА НАЧИНАЕТСЯ ДВИЖЕНИЕ ЗА ЭМАНСИПАЦИЮ ЖЕНЩИН, А С ДРУГОЙ НАРУШАЕТ ЗАПРЕТ НА ИЗОБРАЖЕНИЕ СЕКСУАЛЬНОСТИ, ХАРАКТЕРНЫЙ ДЛЯ ПИСЬМЕННОЙ РУССКОЙ КУЛЬТУРЫ, НАЧИНАЯ С ЕЕ ВИЗАНТИЙСКИХ ИСТОКОВ. КАТЕРИНА ИЗМАЙЛОВА, «ЛЕДИ МАКБЕТ» ЛЕСКОВА, НАХОДИТСЯ ВО ВЛАСТИ ЛЮБОВНОГО ВЛЕЧЕНИЯ, НЕ ЗНАЮЩЕГО ОГРАНИЧЕНИЙ НИ ОБЩЕПРИНЯТОЙ МОРАЛИ, НИ СОЦИАЛЬНЫХ ПРИЛИЧИЙ. ЭТО ВЛЕЧЕНИЕ ТОЛКАЕТ ЕЕ НА СОВЕРШЕНИЕ ВСЕ БОЛЕЕ БЕЗЖАЛОСТНЫХ ПРЕСТУПЛЕНИЙ И ПОСЛЕДНИЕ ДНИ ГЕРОИНИ ПРОЙДУТ ВО МРАКЕ ОДЕРЖИМОСТИ. ОНА БУДЕТ ЖЕСТОКО НАКАЗАНА ПОЗОРНОЙ СМЕРТЬЮ, ЕДИНСТВЕННО ВОЗМОЖНЫМ ВОЗДАЯНИЕМ ЗА ТО МНОГОКРАТНОЕ ПОПРАНИЕ ЧЕЛОВЕЧЕСКИХ ЗАКОНОВ, С КОТОРЫМ СВЯЗАНЫ ЕЕ ПОСТУПКИ.

ОТТАЛКИВАЯСЬ ОТ ЭТОГО ОБРАЗА ЖЕНЩИНЫ, КОТОРОЙ ВЛАДЕЮТ ИСКЛЮЧИТЕЛЬНО СЕКСУАЛЬНЫЕ ИМПУЛЬСЫ - И ОНА СЛЕДОВАТЕЛЬНО ПОДВЕРГАЕТ СЕРЬЕЗНОЙ ОПАСНОСТИ МИР МУЖСКОЙ РАЦИОНАЛЬНОСТИ-, ДМИТРИЙ ШОСТАКОВИЧ СОЗДАЛ ОПЕРУ О СОЦИАЛЬНОМ ОСВОБОЖДЕНИИ И О СОСТРАДАНИИ, ПОДВЕРГНУВ ЗНАЧИТЕЛЬНЫМ ТРАНСФОРМАЦИЯМ ПЕРСОНАЖ КАТЕРИНЫ ИЗМАЙЛОВОЙ: ГЕРОИНЯ ЛЕСКОВА СТАНОВИТСЯ «УБИЙЦЕЙ БЕЗ ВИНЫ», ТРАГИЧЕСКОЙ ЖЕРТВОЙ ТОЙ ЖЕСТОКОСТИ И УГНЕТЕНИЯ, КОТОРЫЕ ХАРАКТЕРИЗОВАЛИ СМЕРТЕЛЬНО ТЯЖЕЛУЮ МОРАЛЬНУЮ И СОЦИАЛЬНУЮ АТМОСФЕРУ «БУРЖУАЗНОЙ И ФЕОДАЛЬНОЙ РОССИИ» ДОРЕВОЛЮЦИОННОЙ ЭПОХИ. ДЛЯ ЛЕСКОВА УБИЙСТВО ВПИСЫВАЛОСЬ В РЯД ПОСЛЕДСТВИЙ СЕКСУАЛЬНОЙ РАСПУЩЕННОСТИ; ДЛЯ ШОСТАКОВИЧА, КОТОРЫЙ НЕ ОТЯГОЩАЕТ НИКАКИМ МОРАЛЬНЫМ ОСУЖДЕНИЕМ ЗАЯВЛЕННОЕ КАТЕРИНОЙ ПРАВО НА СЕКСУАЛЬНОЕ УДОВОЛЬСТВИЕ, УБИЙСТВО - ЭТО ПОРЫВ К ОСВОБОЖДЕНИЮ, А ТАКЖЕ РЕАКЦИЯ НА ИСПЫТАННЫЕ УНИЖЕНИЯ.

ТЕМ НЕ МЕНЕЕ ТВОРЕНИЕ ШОСТАКОВИЧА ГЛУБОКО НЕОДНОЗНАЧНО. ХАРАКТЕРНА ДЛЯ ОПЕРЫ 
КРАЙНЯЯ ЖЕСТОКОСТЬ (КАК В СОЦИАЛЬНЫХ МОТИВАХ, ТАК И В МУЗЫКАЛЬНОЙ ОРГАНИЗАЦИИ), ЭТО НЕ ТОЛЬКО ЖЕСТОКОСТЬ ОТНОШЕНИЙ И ПОВЕДЕНИЯ ЛЮДЕЙ В РУССКОМ ФАЛЛОКРАТИЧЕСКОМ ПАТРИАРХАЛЬНОМ ОБЩЕСТВЕ ВТОРОЙ ПОЛОВИНЫ ХІХ ВЕКА, КОТОРЫЕ РАЗОБЛАЧАЮТСЯ МОЛОДЫМ АВТОРОМ, СТРЕМЯЩИМСЯ ДАТЬ СОВЕТСКИМ ВЛАСТЯМ ДОКАЗАТЕЛЬСТВА СВОЕЙ ЛОЯЛЬНОСТИ. НА САМОМ ДЕЛЕ ХУДОЖЕСТВЕННЫМ ВОПЛОЩЕНИЕМ ОПРЕДЕЛЕННОГО ИСТОРИЧЕСКОГО МОМЕНТА (Т.Е. СТАЛИНСКОЙ ЭПОХИ) ЯВЛЯЕТСЯ КАТЕРИНА ИЗМАЙЛОВА, ПРЕДСТАЮЩАЯ В ОПЕРЕ ЛЕДИ МАКБЕТ МЦЕНСКОГО УЕЗДА В ДВОЙСТВЕННОЙ РОЛИ ЖЕРТВЫ И ПАЛАЧА. ТАКАЯ ДВУСМЫСЛЕННОСТЬ САМИМ ПОДЛИННЫМ ОБРАЗОМ ВЫРАЖАЕТ «ДУХ ВРЕМЕНИ» - КОЛЕБАНИЕ МЕЖДУ СТРАХОМ И ВОСХИЩЕНИЕМ ПЕРЕД ЛИЦОМ НАБИРАЮЩИХ СИЛУ ЖЕСТОКОСТЕЙ ТОТАЛИТАРИЗМА.

INDEX

Mots-clés : Lady Macbeth, Dmitri Chostakovitch, Nikolaï Leskov, opéra russe, littérature russe, sexualité féminine, émancipation

\section{AUTEUR}

CATHERINE GÉRY

INALCO, Centre de recherche Europe-Eurasie 